\title{
Transferência de tecnologias aos produtores rurais: eventos científicos e tecnológicos
}

\section{em tempos de pandemia}

\author{
Technology transfer to farmers: scientific and technological events in times of pandemic \\ Transferencia de tecnología a los agricultores: eventos científicos y tecnológicos en tiempos de \\ pandemia
}

Recebido: 11/11/2021 | Revisado: 17/11/2021 | Aceito: 14/12/2021 | Publicado: 01/01/2022

\author{
Debora Zumkeller Sabonaro \\ ORCID: https://orcid.org/0000-0001-8604-7712 \\ Universidade Federal de São Carlos, Brasil \\ E-mail: dzsabonaro@hotmail.com \\ Luiz Antonio Martinelli \\ ORCID: https://orcid.org/0000-0002-7103-7551 \\ Universidade de São Paulo, Brasil \\ E-mail: lamartinelli50@gmail.com \\ Janaina Braga do Carmo \\ ORCID: https://orcid.org/0000-0002-2871-9880 \\ Universidade Federal de São Carlos, Brasil \\ E-mail: jbcarmo2008@gmail.com
}

\begin{abstract}
Resumo
Este estudo buscou a gestão das informações tecnológicas de eventos agropecuários, de modo a possibilitar a utilização de forma eficiente de transferência de tecnologia, buscando gerar resultados positivos aos produtores rurais através de estudo de casos. A proposta está sendo desenvolvida pelo Programa de Pós-graduação em Biotecnologia e Monitoramento Ambiental, Universidade Federal de São Carlos, Campus Sorocaba, Estado de São Paulo - Brasil. Os procedimentos utilizados, neste trabalho, se caracterizam como pesquisa aplicada. Foram analisadas a transferência de tecnologia através de eventos científicos e Tecnológicos realizados no Projeto: "Gestão de tecnologias geradas em projetos de expansão da cana-de- açúcar e seus efeitos ambientais" contando com o apoio da Fundação de Amparo à Pesquisa do Estado de São Paulo no Processo: 2017/18918-5. Através dos resultados obtidos, pode-se inferir que estas práticas são eficazes, principalmente através de meios digitais, aos produtores rurais, o que afirma a necessidade de incentivar estes processos de transferência de tecnologia. Sugere-se a utilização de meios digitais para divulgação de eventos agropecuários, buscando maior integração do número de produtores rurais, visando ampliar possibilidades de aplicação das tecnologias produzidas e divulgadas. Com isto, seguem as tendências de digitalização, minimizando as dificuldades de acompanhamento dos eventos por parte dos produtores rurais, contribuindo para a ampliação de fronteiras.
\end{abstract}

Palavras-chave: Tecnologia; Produtores rurais; Eventos tecnológicos.

\begin{abstract}
This study sought to manage the technological information of agricultural events, in order to enable the efficient use of technology transfer, seeking to generate positive results for rural producers through case studies. The proposal is being developed by the Graduate Program in Biotechnology and Environmental Monitoring, Federal University of São Carlos, Sorocaba Campus, State of São Paulo- Brazil. The procedures used in this work are characterized as applied research. The transfer of technology was analyzed through scientific and technological events carried out in the Project: "Management of technologies generated in projects for the expansion of sugarcane and its environmental effects" with the support of the São Paulo Research Foundation Paulo in the Process: 2017/18918-5. Through the results obtained, it can be inferred that these practices are effective, mainly through digital media, for rural producers, which affirms the need to encourage these technology transfer processes. It is suggested the use of digital media for the dissemination of agricultural events, seeking greater integration of the number of rural producers, aiming at expanding the possibilities of applying the technologies produced and disseminated. With this, digitalization trends follow, minimizing the difficulties of rural producers in monitoring events, contributing to the expansion of borders.
\end{abstract} Keywords: Technology; Rural producers; Technological events. 


\begin{abstract}
Resumen
Este estudio buscó la gestión de la información tecnológica de los eventos agrícolas, para permitir el uso de la transferencia de tecnología eficiente, buscando generar resultados positivos para los productores rurales a través de estudios de caso. La propuesta está siendo desarrollada por el Programa de Postgrado en Biotecnología y Monitoreo Ambiental de la Universidad Federal de São Carlos, Campus Sorocaba, Estado de São Paulo - Brasil. Los procedimientos utilizados en este trabajo se caracterizan como investigación aplicada. Se analizó la transferencia de tecnología a través de eventos científicos y tecnológicos realizados en el Proyecto: "Gestión de tecnologías generadas en proyectos de expansión de la caña de azúcar y sus efectos ambientales" contando con el apoyo de la Fundación de Apoyo a la Investigación del Estado de São Paulo en el Proceso: 2017/18918-5. A través de los resultados obtenidos, se puede inferir que estas prácticas son efectivas, principalmente a través de los medios digitales, para los productores rurales, lo que afirma la necesidad de fomentar estos procesos de transferencia de tecnología. Se sugiere el uso de medios digitales para la difusión de eventos agropecuarios, buscando una mayor integración del número de productores rurales, buscando ampliar las posibilidades de aplicación de las tecnologías producidas y difundidas. Con esto, sigue las tendencias de digitalización, minimizando las dificultades de seguimiento de los eventos por parte de los productores rurales, contribuyendo a la expansión de las fronteras.
\end{abstract}

Palabras clave: Tecnología; Productores rurales; Eventos tecnológicos.

\title{
1. Introdução
}

As inovações estão correlacionadas com o crescimento da produção agrícola podendo ser correlacionada com o desempenho produtivo. Desta forma, as inovações contribuíram para o enriquecimento no campo e deram maior valor ao papel dos investimentos em tecnologia, uso de conhecimento, aplicação de capital humano e capacidade gerencial (Mendes \& Buainain, 2015).

Em função da pandemia de Covid 19, em função do isolamento, as atividades precisaram sofrer adaptadações, incluindo as atividades extensão e treinamento técnico. As modificações foram realizadas com o ensino remoto, uma forma para que treinamentos técnicos, eventos científicos e tecnológicos de extensão, que antes eram realizados de maneira presencial passassem a ser realizados de forma virtual. Embora os desafios, limitações e adaptações sejam de grande expressão, porém com evoluções fantásticas segundo estudos conduzidos por Vercelli (2020).

Segundo a CNN Brasil (2020), ferramentas como o Google Meet, Microsoft Teams, Skype e Zoom ascenderam o número de usuários após o início da realização de atividades de forma remota. A utilização do Google Meet passou a ser 25 vezes maior no final do mês de março, quando comparada a janeiro, equivalente a um aumento de $60 \%$ por dia. Pode-se verificar um aumento de $70 \%$ para Skype no período.

As inovações diárias na agricultura são impulsionadas pela transformação digital. A aquisição de dados, plantio e colheita em tempo real, aplicativos para gestão de áreas agrícolas, manejo, gestão de defensivos e insumos, adequando ao Código Florestal (Embrapa, 2018).

O destaque da agricultura Brasileira, no mercado externo está relacionado correlacionada a transformação digital, e esta depende de profissionais capacitados para implicação de tecnologias. A internet possibilita o acesso a informações, aplicativos, dentre outros (Brasil, 2018).

A internet é fundamental para as atividades de Transferência de Tecnologia, visto que as informações são oriundas de múltiplas fontes a nível global (Mosso, 2010; Ovtt, 2013).

As tecnologias digitais revolucionam a comunicação, sendo funda, em tais para aproximação de pessoas de diversos locais do mundo. Isso ficou ainda mais em evidência com a pandemia. As redes sociais on-line facilitam a troca de informações de forma instantânea por meio das conexões feitas on-line (Silva \& Leite, 2016).

A transferência de tecnologia (TT) contempla atividades que viabilizam o repasse de conhecimentos, tecnologias, além de aspectos relativos a convenções sociais e interesses econômicos (Brescia et al., 2016). Para que uma tecnologia seja transferida, o agente transferidor deve utilizar mecanismos que viabilizem o processo de transferência (Berbegal et al., 2015; 
Bozeman et al., 2015; Cavalcante et al. 2019). A transferência de tecnologia desenvolve um papel fundamental no processo de adoção de inovações tecnológicas, principalmente quanto à difusão de informações que auxiliem no processo de tomada de decisões para o meio rural (Sabonaro \& Braga 2020).

Este artigo possui como objetivo geral, a proposta de verificar através de estudos de caso, a eficiência da transferência de tecnologia através de eventos agropecuários aos produtores rurais.

\section{Metodologia}

Este estudo apresenta como objetivo central, conhecer os principais aspectos que norteiam a inovação e a transferência de tecnologia. Esta pesquisa foi realizada no Programa de Pós-graduação em Biotecnologia e Monitoramento Ambiental, Universidade Federal de São Carlos, Campus Sorocaba, Estado de São Paulo - Brasil.

Os eventos científicos utilizados neste trabalho, resultaram dos projetos: "Gestão de tecnologias geradas em projetos de expansão da cana-de- açúcar e seus efeitos ambientais" e "Consequências ambientais da conversão pastagem-cana-de-açúcar e intensificação de pastagens”. Estes projetos contam com o apoio da Fundação de Amparo à Pesquisa do Estado de São Paulo nos processos 2017/18918-5 e 2015/18790-3.

Para o estudo de caso foram utilizados quatro eventos Tecnológicos e dois minicursos, realizados durante a vigência do projeto, buscando incentivar o desenvolvimento tecnológico e a transferência de tecnologia ao setor agropecuário: 'Workshop Inovação e transferência de tecnologia'; 'Workshop de Inovações Interdisciplinares para Sustentabilidade', 'Simpósio de Biotecnologia e Engenharia Florestal da UFSCar', 'Treinamento Técnico do Software VS', 'Introdução a propriedade intelectual com foco em patentes' e 'Busca de anterioridades em Tecnologia'.

Segundo Gil (2002) o estudo de caso é um dos meios mais completos quando comparado aos outros. Neste estudo foram utilizados dados de pessoas e dados documentais. Os estudos de caso podem ser constituídos tanto de um único, dois ou vários casos. Justifica-se a utilização de um único quando o caso estudado é único ou extremo (Gil, 2002).

\section{Resultados e Discussão}

\subsection{Identificar as necessidades de informações e seu respectivo público}

Os eventos agropecuários integram produtores rurais, empresários, pesquisadores e organizações. As informações produzidas nestes eventos são fundamentais para a transferência de tecnologia, sendo fundamentais meios eficientes para o repasse de informações.

\subsection{Características dos Eventos utilizados como estudos de caso}

Os eventos 'Workshop de Inovação e transferência de tecnologia'; 'Workshop de Inovações Interdisciplinares para Sustentabilidade' e 'Simpósio de Biotecnologia e Engenharia Florestal da UFSCar' foram eventos realizados presencialmente. Já o evento 'Treinamento Técnico do Software VS foi organizado para ser presencialmente e através de live com disponibilidade via internet. Os minicursos: 'Introdução a propriedade intelectual com foco em patentes' e 'Busca de anterioridades em Tecnologia' foram realizados via google meet.

\subsection{1 'Workshop de Inovação e Transferência de Tecnologia'}

Este evento contou com uma Mesa Redonda: 'Transferência de conhecimento e experiências adquiridas em campo para geração de tecnologias' e três apresentações orais com diferentes profissionais. Além das palestras, o evento contou com uma mesa redonda, afim de uma maior integração entre os participantes do evento científico "Workshop de Inovação e 
Transferência de Tecnologia” ocorrido no Campus da UFSCAR Sorocaba, SP.

\subsection{2 'Workshop de Inovações Interdisciplinares para Sustentabilidade'}

O evento contou com uma Mesa Redonda: 'Projetos Tecnológicos: Tecnologia e transferência de tecnologia para Inovação' e uma apresentação oral da Palestra: ‘A pesquisa científica e/ou tecnológica na Empresa dentro do Programa de Pós Graduação em Biotecnologia e Monitoramento Ambiental', além de apresentações de estudos de casos.

\subsection{3 'Simpósio de Biotecnologia e Engenharia Florestal da UFSCar.}

Neste evento foram apresentadas duas palestras: 'A importância da pesquisa para geração e transferência de novas tecnologias' e 'Uso de técnicas de nucleação em áreas urbanas e rurais'; treinamento técnico na área de Levantamento Topografico e contou ainda com uma mesa redonda ('A importância da pesquisa para geração e transferência de novas tecnologias').

\subsection{4'Treinamento Técnico do Software VS}

A tecnologia foi desenvolvido para orientar os produtores rurais sobre o estado de conservação/ degradação de áreas de preservação permanente e ou/ áreas de nascentes utilizando tecnologia multiplataforma. O evento foi organizado para ser realizado de forma presencial e através de live com disponibilidade via internet, objetivando garantira eficácia da divulgação.

3.2.4 'Introdução a propriedade intelectual com foco em patentes' e 'Busca de anterioridades em Tecnologia' foram realizados via google meet. Atingiu um público mediano por conta da falta de divulgação e nãoficar disponível na internet.

\subsection{Discussão}

O impacto das inovações a favor da difusão tecnológica e modernização, assim como maior lucratividade e produtividade da agricultura brasileira. As tecnologias digitais são fundamentais para o desenvolvimento das atividades cotidianas de plantio, colheita e manejo de animais, softwares para gestão de propriedades, dentre outras atividades. Além da transferência de tecnologia em eventos tecnológicos.

A tecnologia reflete nas aplicações como a maquinas inteligente, sistemas de produção e evolução processos de fabricação e gestão da informação, sendo assim a gestão tecnológica é fortalecida pelo setor biológico, com impacto nos processos produtivos (Hernández, Domínguez e Restrepo, 2009).

Com vasto campo de informações, foi necessário a criação dos métodos de coleta. Estes métodos derivam por exemplo da patentometria, podendo ser classificados como métodos quantitativos (Noronha \& Maricato, 2008). As buscas na internet ou por softwares sobre informações, geram dados que são analisados quantitativamente e consistem sendo importantes para a tomada de decisão. São classificados como métodos quantitativos (Rivero; Sánchez; Suárez, 2009).

Silvina (2018) sugeriu a utilização de um aplicativo que, com várias funções de divulgação de informações, aproximando o produtor rural de vários eventos agropecuários, o que aumentam as possibilidades de transferência de tecnologias. Sesta forma, a digitalização aumentou significamente, aumentando de foram significativa o acesso a divulgação de eventos pelos produtores rurais e auxilia as entidades federais, estaduais na divulgação destes eventos.

As lives não são uma remodelagem, do que já existia. Porém, como é o caso da incorporação de conteúdos acadêmicos, como aulas, palestras e reuniões foram fundamentais para suprir as exigências de abrangência mundial, sendo fundamental para a adaptação do contexto, visto que possuiu ferramentas digitais durante a pandemia se tornou ferramenta para sobrevivência de autônomos, empregadores e empregados (Fettermann et al., 2020). 
A Transferência de Tecnologias está correlacionada à agregação de conhecimentos, visto que, são áreas do processo de inovação da tecnologia (Balbino; Martinez; Galerani, 2011).

Balbino, Martinez e Galerani (2011) descrevem que o processo que no processo de TT existem fatores não tecnológicos que fazem parte do processo, sendo os atores envolvidos; eficiência; avaliação da metodologia e atendimento ou avaliação das necessidades.

A avaliação, os processos e capacitação de atividades executadas na TT são importantes considerações para a sua integração (Balbino, Barcellos, Stone, 2011). Assim, os diferentes profissionais envolvidos permitem a produção de inovações com um foco específico, sendo importante ações diferentes de TT com parte na capacitação continua, tanto na teoria quanto na prática, como os produtores rurais, quanto os técnicos para a adoção de sistemas de integração (Cordeiro et al., 2015).

Pode-se verificar que uma boa divulgação, não se limita apenas a divulgação via site das Universidades, os três primeiros eventos obtiveram concentração do público regional. Os minicursos foram realizados via meet para um público selecionado, mas faltou divulgação para a eficiência. Já o treinamento técnico não se limitará apenas ao público regional, além de funcionar como uma ferramenta de treinamento e divulgação e funcionamento de um software.

\section{Considerações Finais}

Os avanços tecnológicos despertam oportunidades, surgindo necessidades de se trabalhar as adaptações, principalmente em tempos de Pandemia, como acesso às informações e ao conhecimento, difusão das tecnologias, dentre outras.

A ferramenta digital no campo colabora com o desenvolvimento social, econômico e ambiental sendo fundamental pra a integração de diferentes áreas de conhecimento.

A transferência de tecnologia e conhecimentos aos produtores rurais apresentam diferentes abrangências no Brasil, ficando concentrados em alguns Estados do país. Uma das formas do maior acesso à informação tecnológica pelo produtor rural é através da divulgação e disponibilização de eventos via internet.

Os eventos via internet, em tempos de pandemia, aumentaram e sofreram adaptações de diferentes formas e provavelmente se manterá em, buscando construir conhecimentos e de buscar melhorias para a transferência de tecnologias.

Para esta pesquisa, o aumento da transferência de tecnologias aos produtores rurais em colaboração com os centros de pesquisas, universidades e empresas através da divulgação nos eventos agropecuários, lives, dia de campo, dentre outras formas é fundamental como ferramenta eficaz deste processo.

\section{Agradecimentos}

À Fundação de Amparo à Pesquisa do Estado de São Paulo- Processos 2017/18918-5 e 2015/18790-3.

\section{Referências}

Balbino, L. C.; Barcellos, A. O.; Stone, L. F. (Ed.) (2011). Marco Referencial: Marco Referencial: Integração Lavoura- Pecuária-floresta. Brasília, DF: Embrapa, $130 \mathrm{p}$.

Berbegal-Mirabent J, García, J. L. S., \& Ribeiro-Soriano, D. E. (2015). University-industry partnerships for the provision of R\&D services. Journal of Business Research. 15 (68), 1407-1413. 10.1016/j.jbusres.2015.01.023

Bozeman, B; Rimes, H \& Youtie, J (2015). The evolving state-of-the-art in technology transfer research: revisiting the contingent effectiveness model. Research Policy. 15(44), 34-49. 10.1016/j.respol.2014.06.008

Brasil. Ministério da Ciência, Tecnologia, Inovações e Comunicações. Estratégia brasileira para a transformação digital: E-digital. Brasília. <http://www.mctic.gov.br/mctic/export/sites/institucional/estrategiadigital.pdf>.

Brescia, F, Colombo, G., \& Landoni, P (2016). Organizational structures of knowledge transfer offices: an analysis of the world's top-ranked universities. The Journal of Technology Transfer. 16 (41): 132-151. 10.1007/s10961-014-9384-5 
Cavalcante, F. V; Almeida, M. B. C., De; Renault, T. B. (2019). Intervenientes dos processos de transferência tecnológica em uma instituição de ciência e tecnologia: O Caso Fiocruz. Revista Gestão \& Tecnologia. 19 (2), 217-239. <http://revistagt.fpl.edu.br/get/article/view/1383>. https://doi.org/10.20397/21776652/2019.v19i2.1383.

CNN Brasil (2020). Com pandemia, demanda por videoconferência dispara em empresas brasileiras. <https://www.cnnbrasil.com.br/business/2020/04/15/com - pandemia - demanda - por - videoconferencias - dispara - em - empresas - brasileiras >

Cordeiro, L. A. M.; Balbino, L. C; Galerani, P. R; Domit, L. A.; Silva, P. C.; Kluthcouski, J; Vilela, L; Marchão, R. L.; Skorupa, L. A.; Wruck, F. J. (2015). Transferência de tecnologias para adoção da estratégia de integração Lavoura-Pecuária-Floresta. In: CORDEIRO, L. A. M.; Vilela, L; Kluthcouski, J.; Marchão, R. L. (Ed.). Integração Lavoura-Pecuária-Floresta: o produtor pergunta, a Embrapa responde. Brasília, DF: Embrapa, 377-393. (Coleção 500 Perguntas, 500 Respostas).

Embrapa (Empresa Brasileira de Pesquisa Agropecuária) (2008). Visão 2030: O futuro da agricultura brasileira. Embrapa.

Fettermann, J. V.; Benevenuti, C. B.; Tamariz, A. D. R. (2020). Letramentos em processo: Lives como um gênero textual acadêmico a partir da pandemia do COVID-19. Anais do Encontro Virtual de Documentação em Software Livre e Congresso Internacional de Linguagem e Tecnologia Online, 9 (1). <http://www.periodicos.letras.ufmg.br/index.php/anais_linguagem_tecnologia/article/view/17696/1125613809>.

Gil, A. .C (2002). Como elaborar projetos de pesquisas. Atlas.

Hernández, C. N. J.; Domínguez, O. F. C.; Restrepo, L. A. M (2009). Manufactura biológica e inteligente: atributos de la vida aplicados al desarrollo tecnológico. Revista Ingeniería e Investigación, 29 (2), 127-134.

Mendes, C. I. C. \& Buainain, A. M. (2015). Transferência de Tecnologia: Análise além das fronteiras da Embrapa. https://ainfo.cnptia.embrapa.br/digital/bitstream/item/131728/1/tecnologiainformacao.pdf

Mosso, M. J. (2010). Vigilancia tecnológica: aplicación en la seguridad y defensa. Técnica Administrativa, 9 (2).

Noronha, D. P., \& Maricato, J. M. (2008). Estudos métricos da informação: primeiras aproximações. Revista Eletrônica de Biblioteconomia e Ciências da Informação, 13 (1), 116-128.

Rivero, Y. M.; Sánchez, M. V. G.; Suárez, Y. M. (2009). Modelo de evaluación para software que emplean indicadores métricos en la vigilancia científicotecnológica. ACIMED, 20 (6), 125-140.

OVTT (Observatório Virtual de Transferência de Tecnologia) (2013). Vigilância Tecnológica aplicada ao sector TIC: o Observatório Tecnológico do ITI. <http://pt.ovtt.org/destacados/vigilancia-tecnologica-aplicada-ao- sector-tic-o-observatorio-tecnologico-do-iti>.

Sabonaro, D. Z., \& Braga, J. B. A. (2020). Transferência de tecnologia para o produtor rural: um caminho para a sustentabilidade. R. gest. sust. ambient., 9 (n. esp), 120-132.

Silva, M. O., \& Leite, N. C. (2016). Vozes na sala de aula de língua inglesa: uma experiência com os multiletramentos. In: Multiletramentos no ensino de inglês: experiências da escola regular contemporânea. Ouro Preto: Instituto Federal de Minas Gerais.

Silvina, B. S. (2018). Modelo de Vigilância Tecnológica de Eventos Agropecuários para promoção da Transferência de Tecnologias, 147f. Tese apresentada como requisito parcial à obtenção do título de Doutora em Engenharia de Produção, do Programa de Pós-Graduação em Engenharia e Produção, da Universidade Tecnológica Federal do Paraná.

Vercelli, L. de C. A. (2020). Aulas remotas em tempos de covid-19: a percepção de discentes de um programa de mestrado profissional em educação. Revista @ mbienteeducação, 13(2) 47-60. 